\title{
Aplicação do método da espectrofotometria de derivadas na identificação e doseamento simultâneo de sistemas multicomponentes
}

\author{
Luís Roberto Paschoal*, Weverson Alves Ferreira, Mara Régia Dias Prado e \\ Ana Paula Oliveira Vilela
}

Faculdade de Farmácia, Laboratório de Controle de Qualidade Físico-Químico, UNIFENAS

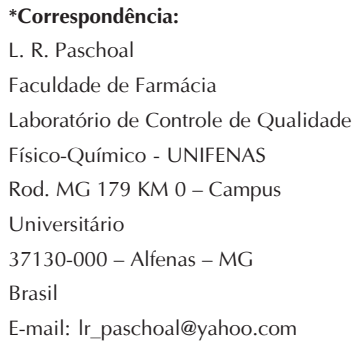

Neste trabalho, demonstrou-se a aplicação do método da espectrofotometria de derivadas no doseamento simultâneo de alguns fármacos em produtos farmacêuticos comerciais. Os métodos desenvolvidos apresentaram-se precisos, sensíveis, seletivos, reprodutivos e de baixo custo.
Unitermos:

- Espectrofotometria de derivadas

- Doseamento simultâneo

- Benzocaína - cloreto de cetilpiridínio

- Lidocaína - prilocaína

- Mebendazol - tiabendazol

\section{INTRODUÇÃO}

A utilização do método da espectrofotometria de derivadas no doseamento simultâneo de dois princípios ativos em diversas formas farmacêuticas tem permitido a obtenção de expressivos resultados em nosso laboratório. Devido às suas propriedades e características, este método apresenta-se como ferramenta muito útil nas análises de fármacos associados em comprimidos, pastilhas, cremes, entre outros, sem a necessidade de separações prévias. A cromatografia líquida de alta eficiência (CLAE) é uma técnica muito explorada, principalmente pelos laboratórios de controle de qualidade das indústrias farmacêuticas, tendo em vista que nos compêndios oficiais, tais como a USP - United States Pharmacopoeia, 2000, a maioria dos fármacos utiliza este método para a separação e posterior doseamento. Entretanto, o método em questão é uma alternativa menos dispendiosa e também eficiente, se comparada ao método da CLAE.

Como prova desse fato, muitos trabalhos recentes (Yesilada et al., 1991; Bonazzi et al., 1996; Erk, Onur, 1997; Hassouna, 1997; Karali et al., 1998; Dinc, Onur, 1998; Paschoal, Ferreira, 2000; Prado et al., 2001,) foram publicados, empregando o método da espectrofotometria de derivadas, que se mostrou ser preciso, sensível, seletivo, reprodutivo e de baixo custo.

\section{ALGUNS ASPECTOS TEÓRICOS DA ESPECTROFOTOMETRIA DE DERIVADAS}

Um dos problemas da espectrofotometria nas regiões do ultravioleta - visível (UV-vis) de misturas de componentes está relacionado com a sobreposição das bandas (overlapping) de transição eletrônica ou de transferência de carga em um mesmo solvente. A Figura 1 ilustra a sobreposição de dois fármacos associados na forma farmacêutica de pastilha e estudada em solução hidroalcoólica por Paschoal e Ferreira (2000).

A diferenciação do espectro de ordem zero não aumenta as informações do espectro original, mas ocorre supressão das bandas largas, realce das bandas estreitas, a resolução de um "ombro" para uma banda, etc., obedecendo, ainda, a Lei de Beer-Lambert.

Em um espectro de primeira ordem (derivada primeira) ocorre a anulação no ponto referente ao comprimento de onda máximo $\left(\lambda_{\max }\right)$ do espectro de ordem zero. É positivo onde a absorção aumenta e negativo onde ela diminui. Assim, uma curva diferencial de ordem $n$ se anula $n$ vezes, apresentando $n+1$ bandas.

Na sobreposição de espectros de derivadas dos componentes de uma mistura, obtidos desse modo, pode-se individualizar melhor os constituintes e até eliminar a interferência de um componente sobre o outro, excipientes, 
entre outros. A Figura 2 mostra os espectros de primeira ordem dos compostos obtidos a partir da Figura 1.

Observa-se nas curvas da Figura 2 que, em $\operatorname{certos} \lambda$, ocorre a anulação do espectro de um componente enquanto há absorção do outro. Deste modo, sobrepondo-se várias derivadas de concentrações crescentes de cada com- ponente e, obtendo-se os valores das amplitudes nos pontos de anulação (zero crossing), é possível construir-se curvas de calibração, além de se eliminar eletronicamente os erros sistemáticos provenientes de interferências (overlapping). Comercialmente, espectrofotômetros que realizam varredura de espectro, apresentam no software de

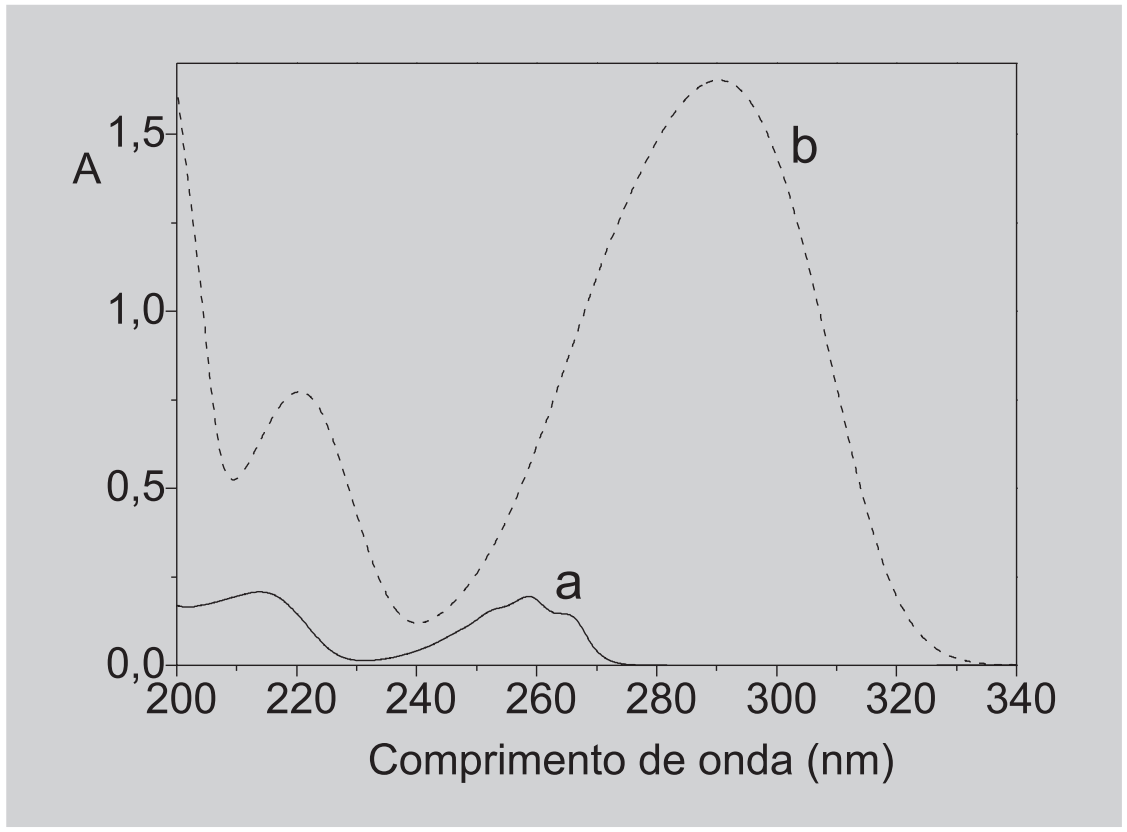

FIGURA 1 - Espectros de absorção de ordem zero do cloreto de cetilpiridínio $16 \mathrm{mg} \mathrm{L}^{-1}$ (a) e da benzocaína $15 \mathrm{mg} \mathrm{L}^{-1}$ (b), obtidos em álcool/água (1:1).

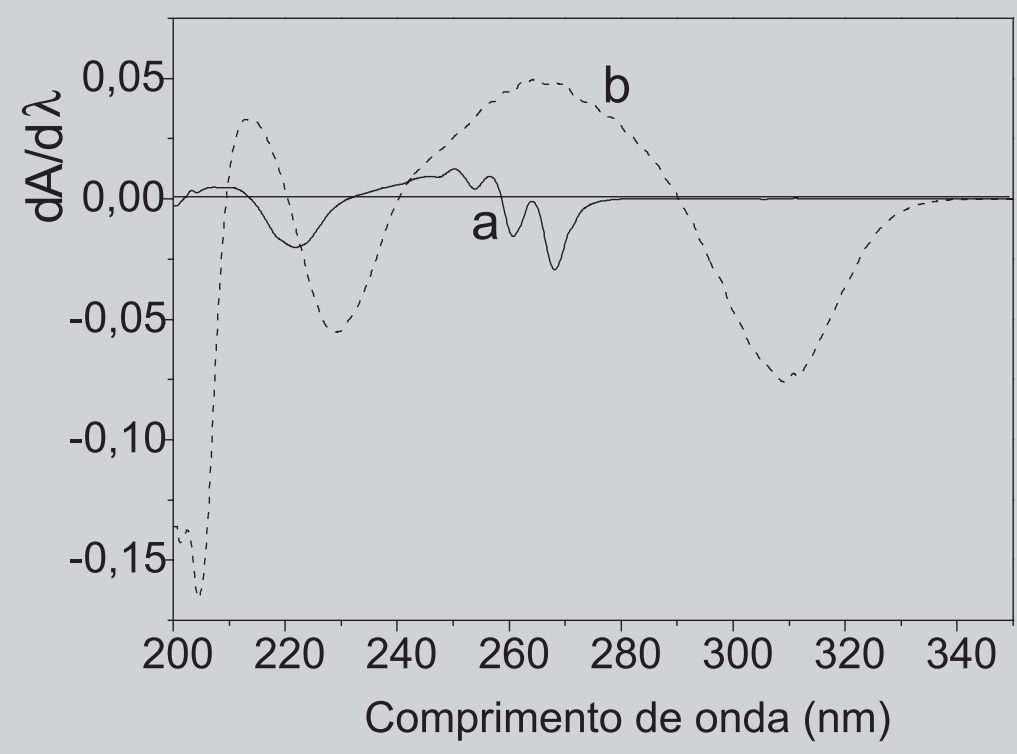

FIGURA 2 - Espectros de absorção de derivada primeira do cloreto de cetilpiridínio $16 \mathrm{mg} \mathrm{L}^{-1}$ (a) e da benzocaína $15 \mathrm{mg} \mathrm{L}^{-1}(\mathrm{~b})$, obtidos em álcool/água (1:1). 
interface, que é um programa que controla o funcionamento do aparelho, a possibilidade de se derivar até a quarta ou mais derivadas, além de outros diferentes recursos necessários à obtenção dessas curvas, tais como $\Delta \lambda$.

Desta forma, os objetivos do presente trabalho são: (a) apresentar a metodologia empregada em nosso laboratório para o doseamento simultâneo de alguns fármacos em diferentes formas farmacêuticas; (b) incentivar alunos de pós-graduação, pesquisadores, laboratórios, entre outros, a utilizarem este método como alternativa eficiente na identificação e análise de misturas de componentes.

\section{MATERIAL E MÉTODOS}

\section{Reagentes}

As matérias-primas cloreto de cetilpiridínio, benzocaína, mebendazol e tiabendazol, foram adquiridas dos distribuidores Henrifarma, Synth, Emellffar Comercial e do Brasil. Os insumos prilocaína e lidocaína, grau USP foram fornecidos pela empresa Cristália - Produtos Químicos Farmacêuticos Ltda.

Já as amostras dos produtos acabados foram aquelas solicitadas aos fabricantes de produtos comerciais acabados e conforme demonstrado abaixo:

1 - Pastilhas Cetil Drops (Luper): cada pastilha contém:

- cloreto de cetilpiridínio $=2,5 \mathrm{mg}$

- benzocaína $=2,0 \mathrm{mg}$

2 - Creme Medicaína $5 \%$ (Cristália):

- lidocaína $=25 \mathrm{mg}$

- prilocaína $=25 \mathrm{mg}$

3 - Comprimidos Octelmin ( Schering-Plough): cada comprimido contém:

- mebendazol $=100 \mathrm{mg}$

- tiabendazol $=166 \mathrm{mg}$

Todos os demais reagentes e solventes empregados foram de grau analítico, com pureza superior a $98 \% \mathrm{v} / \mathrm{v}$.

Toda a água utilizada nas lavagens de vidraria, preparo das soluções e diluições foi recém-destilada e desionizada.

Triplicatas das amostras identificadas por fabricante foram analisadas frente a padrões analíticos e através dos métodos de espectroscopias nas regiões do ultravioleta e infravermelho, análise térmica (TG/DTG), Karl Fischer, ponto de fusão, entre outros.

Os fármacos foram utilizados como adquiridos dos fabricantes, sem purificações prévias.

\section{Soluções Padrão}

Em todas as determinações usualmente preparam-se soluções padrão mais concentradas (estoque) dos fármacos, no solvente determinado, em que ocorreu sua máxima solubilidade. Exemplos:

Solução estoque:

- solução hidroalcóolica $1: 1 \mathrm{a} 100 \mathrm{mg} \cdot \mathrm{mL}^{-1}$ de cloreto de cetilpiridínio ou de benzocaína;

- solução 1:1 (ácido clorídrido e água) $100 \mathrm{mg} \cdot \mathrm{mL}^{-1} \mathrm{de}$ mebendazol ou de tiabendazol;

- solução hidroalcoólica 1:1 a $100 \mathrm{mg} \cdot \mathrm{mL}^{-1}$ de lidocaína ou prilocaína.

A seguir, são preparadas soluções diluídas de concentrações variadas, a partir das soluções estoque correspondentes, em determinada faixa de concentrações, que seguem a Lei de Lambert-Beer, para obtenção dos espectros de ordem zero.

As soluções das amostras de produtos acabados são preparadas do mesmo modo e diluição, sendo, também, obtidos seus espectros de ordem zero.

\section{TÉCNICA EXPERIMENTAL E EQUIPAMENTO UTILIZADO}

Os espectros de ordem zero e suas respectivas derivadas são obtidos na região do ultravioleta (UV), empregando-se espectrofotômetro Shimadzu mod. UV-1601 PC, de varredura, que apresenta as seguintes características: duplo feixe entre 190 - $1100 \mathrm{~nm}$, banda espectral de $2 \mathrm{~nm}$, interfaceado por software, que permite correção automática de linha base e manipulação total do espectro. As análises espectrofotométricas são realizadas a $20^{\circ} \mathrm{C}$, utilizando controlador de temperatura Shimadzu TCC-240 A.

\section{TRATAMENTO DOS DADOS EXPERIMENTAIS}

\section{Espectroscopia eletrônica na região do ultravioleta}

Os espectros de absorção de ordem zero dos fármacos e suas respectivas derivadas são obtidos em solvente adequado, através do estudo da absorção da radiação na região do ultravioleta ( 200 a $350 \mathrm{~nm}$ ) e $\Delta \lambda=2,0 \mathrm{~nm}$. As medidas espectrofotométricas são realizadas em cubetas padronizadas de quartzo de $1 \mathrm{~cm}$ e velocidade de varredura de $8,3 \mathrm{~nm} \mathrm{~s}^{-1}$ ("middle"), observando-se a estabilidade das soluções.

Os máximos de absorção, $\lambda_{\max }$, são, então, identificados empregando-se os recursos do software do espectrofotômetro. Os coeficientes de absortividades molares (e) 
dos compostos são calculados empregando-se a Lei de Lambert-Beer e corrigindo a linha de base obtida para o solvente utilizado.

A seguir, os espectros de derivada primeira são calculados e visualizados a partir dos seus espectros de ordem zero usando-se o software do equipamento. Derivadas superiores são conseguidas com o mesmo procedimento; porém há a necessidade de efetuar-se um ajuste nas curvas através da escolha do valor $\Delta \lambda$ adequado para a melhor relação sinal/ruído.

\section{Preparação das curvas calibração}

A partir da técnica de anulação (zero crossing), isto é, lendo-se as amplitudes de absorção de um componente no comprimento de onda de anulação do outro, são construídas curvas de calibração com a finalidade de se determinar a linearidade do método e permitir a análise quantitativa do produto acabado. A Figura 3 mostra exemplos de curvas de calibração obtida por Paschoal e Ferreira, (2000), para o caso os fármacos ilustrados na Figura 2.

A Tabela I apresenta os resultados obtidos para os teores de cloreto de cetilpiridinio e benzocaína determinados em pastilhas, pelo método de espectrofotometria de derivadas.

Da mesma forma, foi desenvolvida a determinação simultânea de lidocaína e prilocaína em cremes pelo método da espectrofotometria de derivadas (Prado et al., 2001).
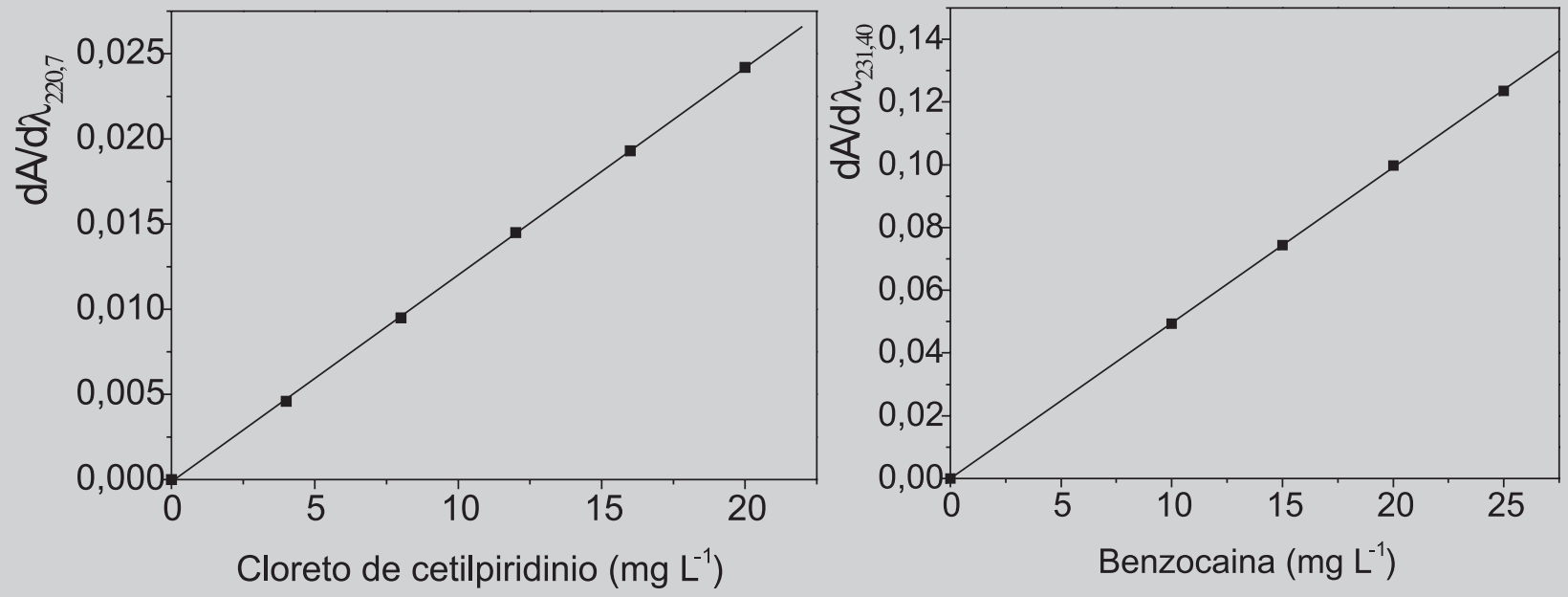

FIGURA 3 - (esquerda) Curva de calibração do cloreto de cetilpiridínio obtida em 220,7 nm (amplitude negativa). A = $1,33 \cdot 10^{-4}+1,22 \cdot 10^{-3} \cdot \mathrm{c}\left(\mathrm{mg} \mathrm{L}^{-1}\right), \mathrm{com} \mathrm{r}=0,9999$. (direita) Curva de calibração da benzocaína obtida em 231,4 nm (amplitude negativa). $\mathrm{A}=3,51 \cdot 10^{-5}+3,37 \cdot 10^{-3} \cdot \mathrm{c}\left(\mathrm{mg} \mathrm{L}^{-1}\right), \mathrm{com} \mathrm{r}=0,9999$.

TABELA I - Valores encontrados para benzocaína e cloreto de cetilpiridínio em misturas binárias, para produto comercial (pastilhas) e para uma simulação do produto em laboratório

\begin{tabular}{|c|c|c|c|c|c|c|c|c|}
\hline \multirow[b]{2}{*}{ Fármaco } & \multicolumn{4}{|c|}{ Simulação $^{\text {(a) }}$} & \multicolumn{4}{|c|}{ Pastilhas ${ }^{(a)}$} \\
\hline & $\begin{array}{l}\text { Valor } \\
\text { teórico } \\
(\mathrm{mg})\end{array}$ & $\begin{array}{l}\text { Quantidade } \\
\text { total encon- } \\
\text { trada }(\mathrm{mg})\end{array}$ & $\begin{array}{l}\text { Recupe- } \\
\text { rado } \\
(\%)\end{array}$ & $\begin{array}{c}\text { RSD } \\
(\%)\end{array}$ & $\begin{array}{l}\text { Valor } \\
\text { teórico } \\
(\mathrm{mg})\end{array}$ & $\begin{array}{l}\text { Quantidade } \\
\text { total encon- } \\
\text { trada }(\mathrm{mg})\end{array}$ & $\begin{array}{l}\text { Recupe- } \\
\text { rado } \\
(\%)\end{array}$ & $\begin{array}{l}\text { RSD } \\
(\%)\end{array}$ \\
\hline Benzocaína & 2,00 & 1,95 & 97,50 & 0,44 & 2,00 & 2,20 & 110,00 & 0,24 \\
\hline $\begin{array}{l}1 \mathrm{D}_{231,40 \mathrm{~nm}} \\
\text { Benzocaína }\end{array}$ & 2,00 & 1,93 & 96,50 & 0,28 & 2,00 & 2,00 & 100,01 & 0,52 \\
\hline $\begin{array}{l}1 \mathrm{D}_{310,00 \mathrm{~nm}} \\
\text { Cloreto de Cetilpiridínio } \\
1 \mathrm{D}_{220,70 \mathrm{~nm}}\end{array}$ & 2,50 & 2,56 & 102,40 & 1,15 & 2,50 & 2,42 & 96,80 & 1,31 \\
\hline
\end{tabular}

(a) os valores são devidos a quatro repetições. 
A Figura 4 apresenta os espectros de ordem zero obtidos para os anestésicos isoladamente.

A seguir são apresentadas as curvas de derivada primeira (Figura 5) e segunda (Figura 6), obtidas a partir dos espectros de ordem zero, evidenciando os comprimentos de onda de anulação dos ativos, do creme e simulação de um creme (Figura 7).
A Tabela II apresenta os resultados encontrados para os anestésicos estudados em creme a $5 \%(\mathrm{~m} / \mathrm{m})$, empregando-se o método da espectrofotometria de derivadas e comparando os resultados com aqueles determinados por CLAE pela indústria fabricante.

A seguir, são apresentados os resultados da determinação simultânea de mebendazol e tiabendazol em com-

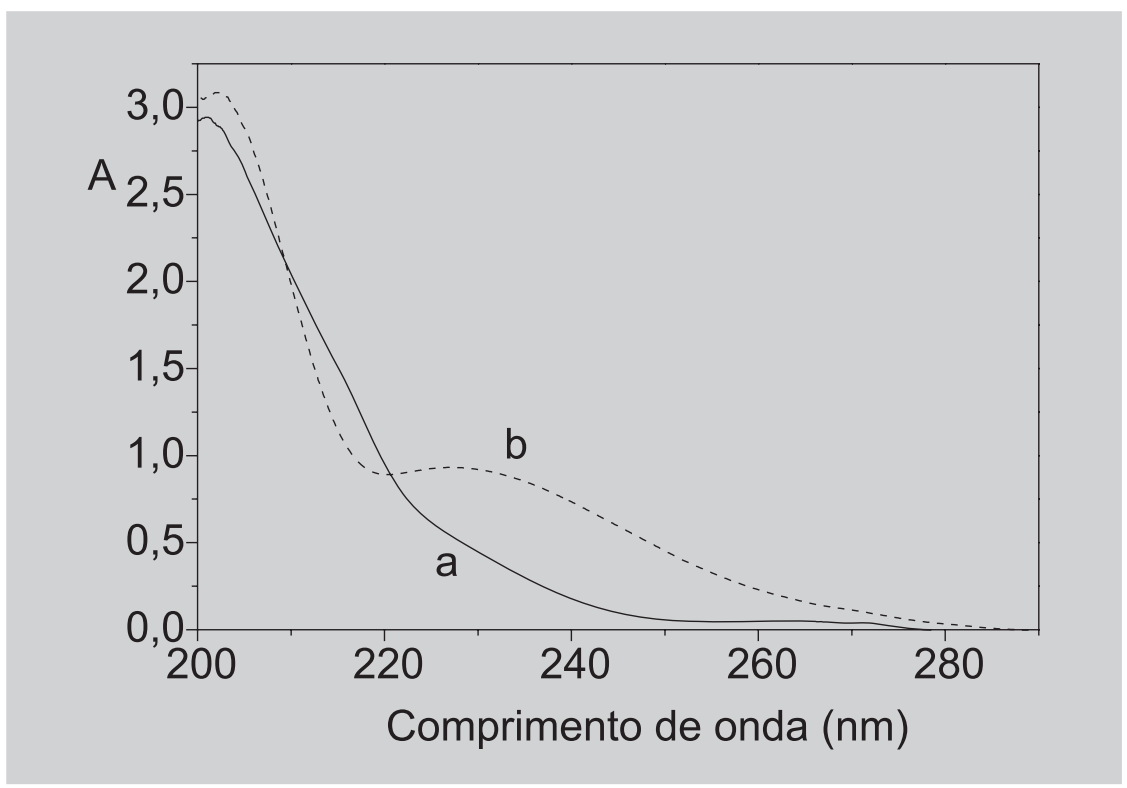

FIGURA 4 - Espectros de aborção de ordem zero da lidocaína $30 \mathrm{mg} \mathrm{L}^{-1}$ (a) e prilocaína $30 \mathrm{mg} \mathrm{L}^{-1}$ (b), obtidos em solução água/álcool 1:1 (v/v).

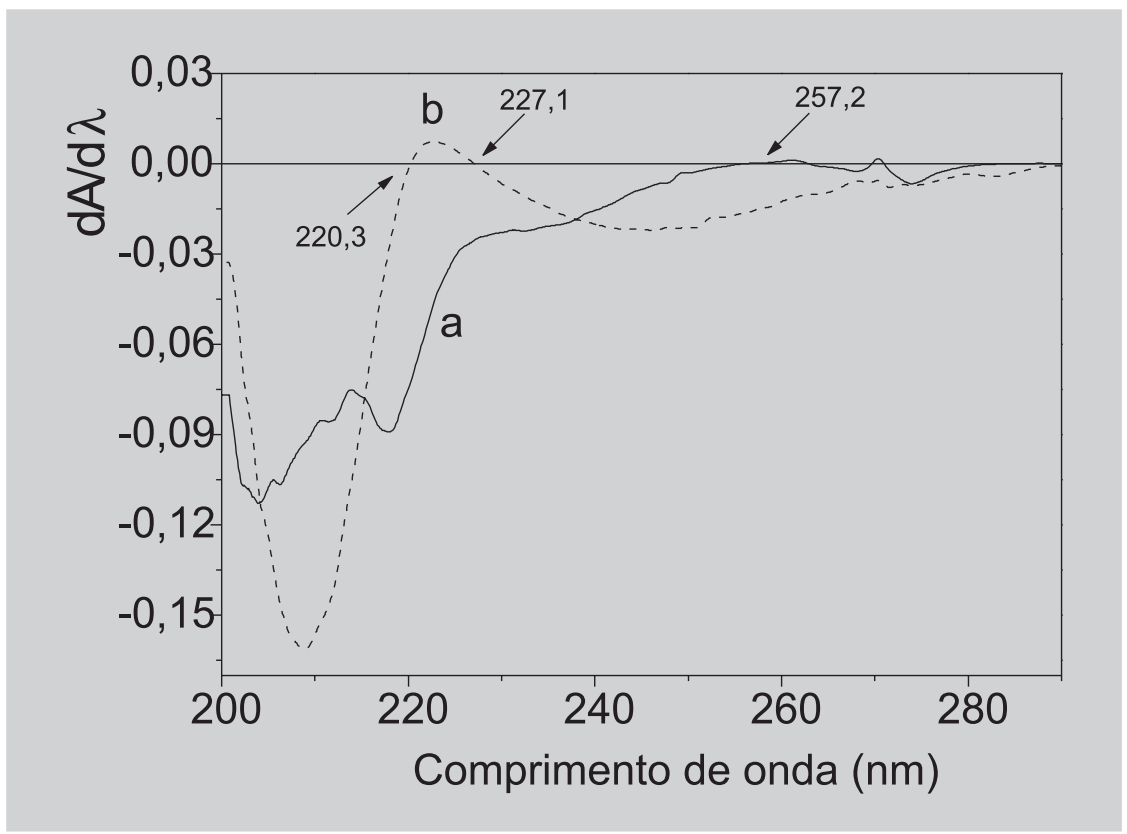

FIGURA 5 - Espectros de absorção de primeira derivada de lidocaína $30 \mathrm{mg} \mathrm{L}^{-1}$ (a) e de prilocaína $30 \mathrm{mg} \mathrm{L}^{-1}$ (b), obtidos em álcool/água (1:1). 


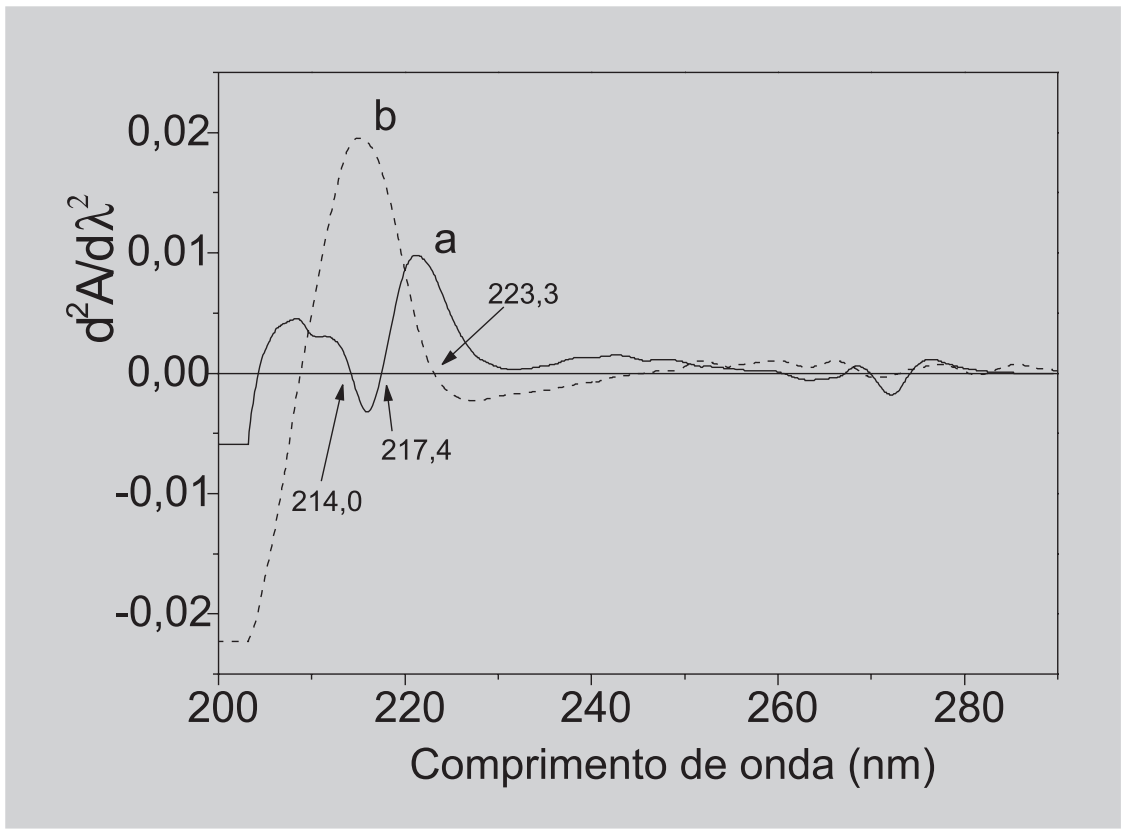

FIGURA 6 - Espectros de absorção de segunda derivada de lidocaína $30 \mathrm{mg} \mathrm{L}^{-1}$ (a) e de prilocaína $30 \mathrm{mg} \mathrm{L}^{-1}$ (b) , obtidos em álcool/água (1:1).

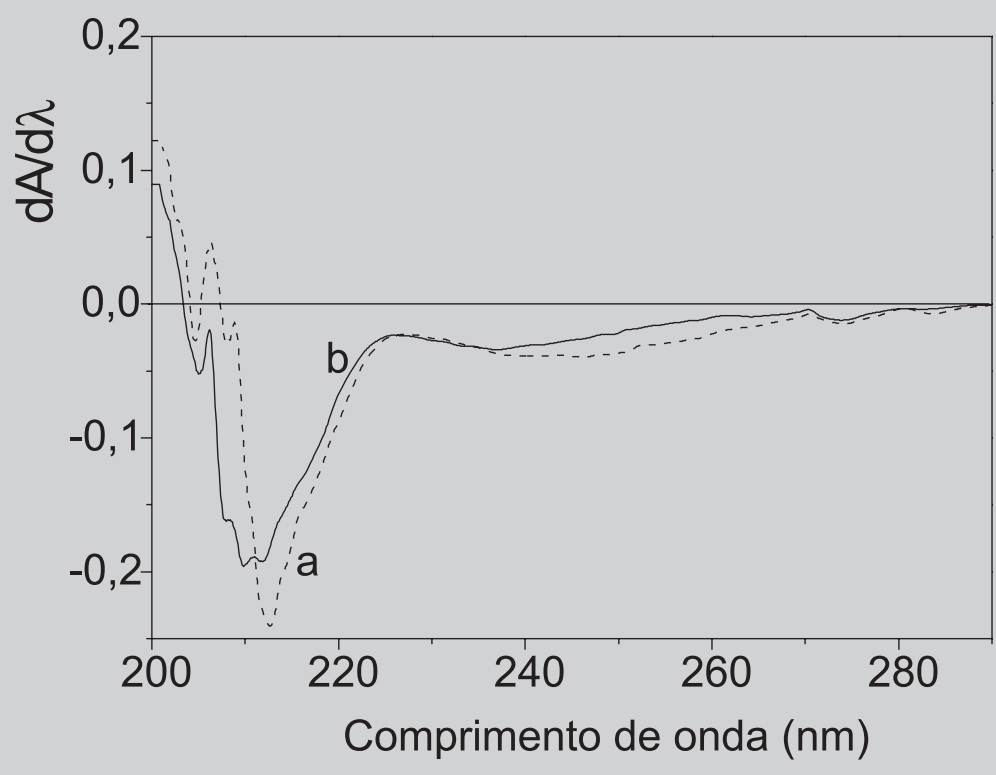

FIGURA 7 - Espectros de primeira derivada do creme (a) e da simulação do creme (b) de lidocaína $30 \mathrm{mg} \mathrm{L}^{-1}$ e prilocaína $30 \mathrm{mg} \mathrm{L}^{-1}$.

primidos através de espectrofotometria de derivadas.

A Figura 8 apresenta os espectros de absorção de ordem zero (impedindo o doseamento convencional) e a Figura 9 os de derivada primeira obtidos dos antihelmínticos mebendazol e tiabendazol em solução ácida, demonstrando a sobreposição das bandas (overlapping).

Pelas curvas obtidas em primeira derivada, observa- se a possibilidade do doseamento simultâneo dos fármacos associados em comprimidos através da técnica de zero-crossing. Assim, foram obtidas as equações de regressão linear correspondentes aos comprimentos de onda de anulação do mebendazol $(287,10 \mathrm{~nm})$ e do tiabendazol $(242,5,00 \mathrm{~nm})$, obtendo $\mathrm{r}=0,9999$ e $\mathrm{r}=$ 0,9993 , respectivamente. 
TABELA II - Teores de lidocaína e prilocaína encontrados na amostras do creme Medicaína 5\%

\begin{tabular}{lcccc}
\hline COMPOSTOS & \multicolumn{2}{c}{ CLAE $^{*}$} & \multicolumn{2}{c}{ ESPECTROFOTOMETRIA DE DERIVADAS** $^{*}$} \\
\hline & $(\mathrm{mg} / \mathrm{g})$ & $\%$ & $\begin{array}{c}{ }^{1} \mathrm{D}_{\lambda=227,1 \mathrm{~nm}} \\
(\mathrm{mg} / \mathrm{g})\end{array}$ & $\begin{array}{c}{ }^{2} \mathrm{D}_{\lambda=217,4 \mathrm{~nm}} \\
(\mathrm{mg} / \mathrm{g})\end{array}$ \\
Lidocaína & $23,75-26,25$ & $95-105$ & 22,96 & $-26,53$ \\
Prilocaína & $23,75-26,25$ & $95-105$ & $n$ & 26,53 \\
\hline
\end{tabular}

*TEORES MÉDIOS: Fonte: Cristália Produtos Químicos Farmacêuticos Ltda.; ** Lote analisado

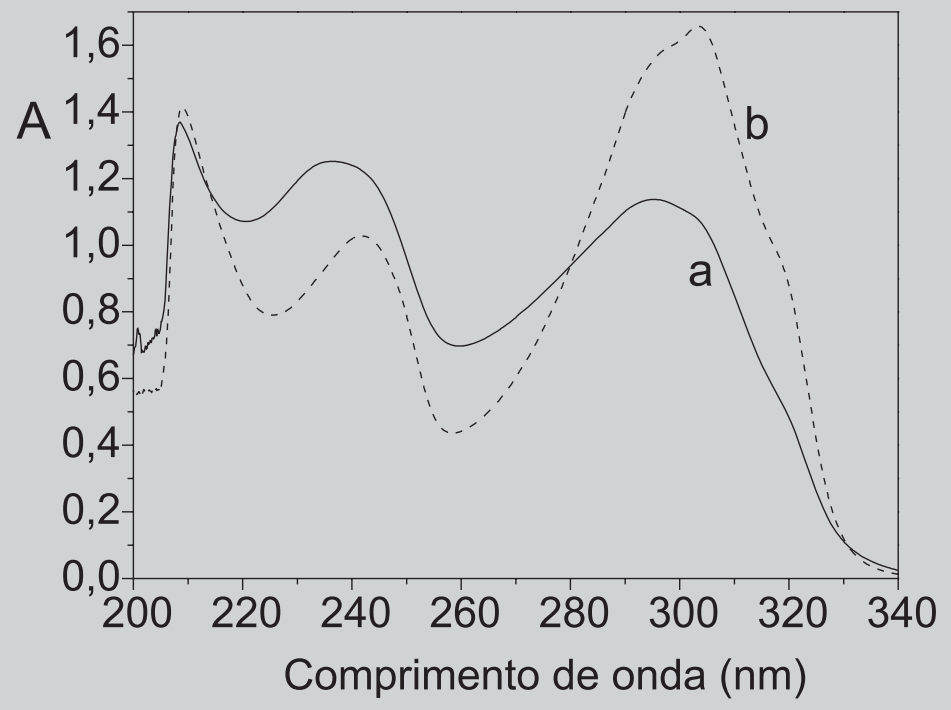

FIGURA 8 - Espectros de absorção de ordem zero de mebendazol (12mg L $\left.\mathrm{m}^{-1}\right)$ (a) e tiabendazol (b) (12 mg L $\left.\mathrm{m}^{-1}\right)$, obtidos em $\mathrm{H}_{2} \mathrm{O} / \mathrm{HCl} 50 \%(\mathrm{v} / \mathrm{v})$.

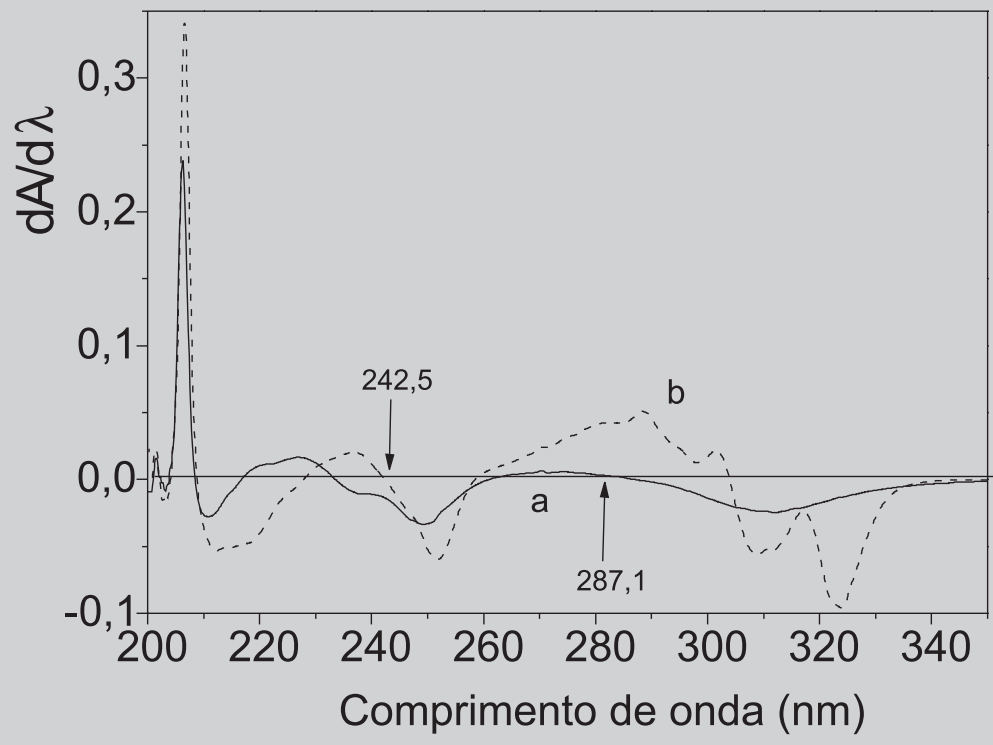

FIGURA 9 - Espectros de absorção de primeira derivada de mebendazol (12 $\left.\mathrm{mg} \mathrm{L}^{-1}\right)$ (a) e tiabendazol (b) (12 $\left.\mathrm{mg} \mathrm{L}^{-1}\right)$, obtidos em $\mathrm{H}_{2} \mathrm{O} / \mathrm{HCl} 50 \%$ (v/v). 
TABELA III - Valores encontrados para tiabendazol e mebendazol em misturas binárias, para produto comercial (comprimidos) e para uma simulação do produto em laboratório

\begin{tabular}{lcccccccc}
\hline & \multicolumn{4}{c}{ Simulação $^{(\mathrm{a})}$} & \multicolumn{4}{c}{ Comprimidos $^{(\mathrm{a})}$} \\
Fármaco & $\begin{array}{c}\text { Valor } \\
\text { teórico } \\
(\mathrm{mg})\end{array}$ & $\begin{array}{c}\text { Quantidade } \\
\text { total encon- } \\
\text { trada }(\mathrm{mg})\end{array}$ & $\begin{array}{c}\text { Recupe- } \\
\text { rado } \\
(\%)\end{array}$ & $\begin{array}{c}\text { RSD } \\
(\%)\end{array}$ & $\begin{array}{c}\text { Valor } \\
\text { teórico } \\
(\mathrm{mg})\end{array}$ & $\begin{array}{c}\text { Quantidade } \\
\text { total encon- } \\
\text { trada }(\mathrm{mg})\end{array}$ & $\begin{array}{c}\text { Recupe- } \\
\text { rado } \\
(\%)\end{array}$ & $\begin{array}{c}\text { RSD } \\
(\%)\end{array}$ \\
\hline Tiabendazol 1D \\
Mebendazol 1D $_{242,50 \mathrm{~mm}}$ & 166,00 & 165,97 & 99,98 & 0,66 & 166,00 & 163,32 & 98,38 & 0,65 \\
\hline
\end{tabular}

(a) os valores são devidos à três repetições.

\section{CONCLUSÕES}

A metodologia proposta neste trabalho configura-se, assim, em alternativa extremamente vantajosa e viável para a solução de problemas espectrais de sobreposição de mais de um componente.

A grande vantagem da utilização da espectrofotometria de derivadas está relacionada com a possibilidade de se minimizar os problemas com interferentes, sem complicados procedimentos de separação ou extração prévios, bem como não necessitar de padrões ultrapuros exigidos na CLAE, além da grande possibilidade da validação do método no doseamento simultâneo, aliada ao baixo preço.

\section{ABSTRACT \\ Application of derivative spectrophotometric method in identification and simultaneous analysis of multi-component system}

The application of the derivative spectrophotometric method in the simultaneous determinations of some drugs in commercial pharmaceutical products is demonstrated in this work. The developed methods proved to be selective, reproductive and of low cost.

UNITERMS: Derivative spectrophotometric methods. Simultaneous determinations. Benzocaine cetylpiridinium chloride. Lidocaine-prilocaine. Mebendazole - thiabendazole.

\section{AGRADECIMENTOS}

A FAPEMIG e ao CNPq, pelo apoio financeiro referente à aquisição do espectrofotômetro de varredura e bolsas de iniciação científica, respectivamente.

As indústrias Cristália - Produtos Químicos Farmacêuticos e a Schering-Plough, pelo fornecimento de padrões bem como de produtos comerciais de suas linhas.

\section{REFERÊNCIAS BIBLIOGRÁFICAS}

BONAZZI, D.; GOTTI, R.; ANDRISANO, V.; CAVRINI, V. Derivative UV determination of atenolol and metroprolol in single and multi-component pharmaceutical dosage forms. Farmaco, Amsterdam, v. 51, n.11, p. 733-738, 1996.

DINC, E.; ONUR, F. Application of a new spectrophotometric method for the analysis of a ternary mixture containing metamidazol, paracetamol and caffeine in tablets. Anal. Chim. Acta, Amsterdam, v.359, p. 93-106, 1998.

ERK, N.; ONUR, F. Simultaneous Determination of Analgine and Paracetamol in Tablet by Spectrophotometric Methods. Anal. Lett., Cork, v.30, n. 6, p. 12011210, 1997

HASSOUNA, M. E. M. Simultaneous Spectreophotometric Determination of Sulfamethoxazole and Trimethoprim in Drugs. Anal. Lett., Cork, v.30, n.13, p. 2341-2352, 1997.

KARALI, S.; OZKIRIMLI, S.; GURSOY, A. Simultaneous determination of medazepam and hyoscine butylbromide in tablets by second-derivative ultraviolet spectrometry. Farmaco, Amsterdam, v.53, p. 62-64, 1998.

PASCHOAL, L. R.; FERREIRA, W. A. Simultaneous determination of benzocaine and cetylpiridinium chloride in tablets by first-derivative spectrophotometric method. Farmaco, Amsterdam, v. 55, p. 687 -693, 2000.

PRADO, M. R. D.; PASCHOAL, L. R.; FERREIRA, W. A.; VILELA, A. P.O . Determinação simultânea de lidocaína e prilocaína em cremes por espectrofotometria de derivadas. Rev. Bras. Cienc. Farm., São Paulo. v. 37, supl.2, p. 74-74. 
THE UNITED STATES PHARMACOPEIA AND THE NATIONAL FORMULARY, US Pharmacopoeial Convention, Rockville, MD, 2000. 2569p.
YESILADA, A.; ERDOGAN, H.; ERTAN, M. 2nd Derivative Spectrophotometric Determination of Paraaminophenol in the Presence of Paracetamol. Anal. Lett., New York, v. 24, n.1, p. 129-138. 1991.

Recebido para publicação em 21 de junho de 2002. 